\title{
A sensitive EUV Schwarzschild microscope for plasma studies with sub-micrometer resolution
}

U. Zastrau, C. Rödel, M. Nakatsutsumi, T. Feigl, K. Appel, B. Chen, T. Döppner, T. Fennel, T. Fiedler, L. B. Fletcher, E. Förster, E. Gamboa, D. O. Gericke, S. Göde, C. Grote-Fortmann, V. Hilbert, L. Kazak, T. Laarmann, H. J. Lee, P. Mabey, F. Martinez, K.-H. Meiwes-Broer, H. Pauer, M. Perske, A. Przystawik, S. Roling, S. Skruszewicz, M. Shihab, J. Tiggesbäumker, S. Toleikis, M. Wünsche, H. Zacharias, S. H. Glenzer, and G. Gregori

Citation: Review of Scientific Instruments 89, 023703 (2018); doi: 10.1063/1.5007950

View online: https://doi.org/10.1063/1.5007950

View Table of Contents: http://aip.scitation.org/toc/rsi/89/2

Published by the American Institute of Physics

\section{Articles you may be interested in}

Nanometer-scale characterization of laser-driven compression, shocks, and phase transitions, by x-ray scattering using free electron lasers

Physics of Plasmas 24, 102709 (2017); 10.1063/1.5008289

Simultaneous $8.2 \mathrm{keV}$ phase-contrast imaging and $24.6 \mathrm{keV}$ X-ray diffraction from shock-compressed matter at the LCLS

Applied Physics Letters 112, 221907 (2018); 10.1063/1.5031907

High repetition rate, multi-MeV proton source from cryogenic hydrogen jets

Applied Physics Letters 111, 114102 (2017); 10.1063/1.4990487

A novel multiplex absorption spectrometer for time-resolved studies

Review of Scientific Instruments 89, 024101 (2018); 10.1063/1.5006539

Setup for meV-resolution inelastic X-ray scattering measurements and X-ray diffraction at the Matter in Extreme Conditions endstation at the Linac Coherent Light Source

Review of Scientific Instruments 89, 10F104 (2018); 10.1063/1.5039329

X-ray spectrometer having 12000 resolving power at $8 \mathrm{keV}$ energy

Review of Scientific Instruments 88, 103107 (2017); 10.1063/1.4999995

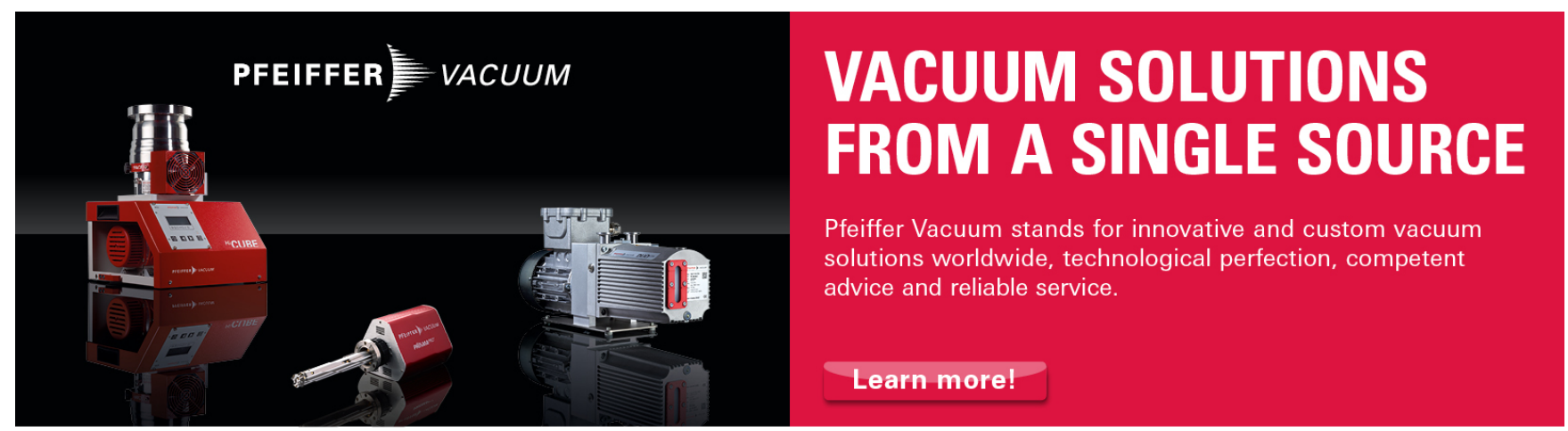




\title{
A sensitive EUV Schwarzschild microscope for plasma studies with sub-micrometer resolution
}

\author{
U. Zastrau, ${ }^{1, a)}$ C. Rödel, ${ }^{2,3,4}$ M. Nakatsutsumi, ${ }^{1}$ T. Feigl, ${ }^{5}$ K. Appel, ${ }^{1}$ B. Chen, ${ }^{6}$ T. Döppner, ${ }^{7}$ \\ T. Fennel, ${ }^{8}$ T. Fiedler, ${ }^{5}$ L. B. Fletcher, ${ }^{3}$ E. Förster, ${ }^{2,4}$ E. Gamboa, ${ }^{3}$ D. O. Gericke, ${ }^{9}$ S. Göde, ${ }^{1}$ \\ C. Grote-Fortmann, ${ }^{1}$ V. Hilbert, ${ }^{10}$ L. Kazak, ${ }^{8}$ T. Laarmann, ${ }^{11}$ H. J. Lee,${ }^{3}$ P. Mabey, ${ }^{12}$ \\ F. Martinez, ${ }^{8}$ K.-H. Meiwes-Broer,${ }^{8}$ H. Pauer, ${ }^{5}$ M. Perske, ${ }^{5}$ A. Przystawik, ${ }^{13}$ S. Roling, ${ }^{14}$ \\ S. Skruszewicz, ${ }^{2,4}$ M. Shihab, ${ }^{8,15}$ J. Tiggesbäumker, ${ }^{8}$ S. Toleikis, ${ }^{13}$ M. Wünsche,,$^{2,4}$ \\ H. Zacharias, ${ }^{14}$ S. H. Glenzer, ${ }^{3}$ and G. Gregori ${ }^{12}$ \\ ${ }^{1}$ European XFEL, Holzkoppel 4, 22869 Schenefeld, Germany \\ ${ }^{2}$ Institute of Optics and Quantum Electronics, Friedrich-Schiller University Jena, Max-Wien-Platz 1, 07743 Jena, \\ Germany \\ ${ }^{3}$ Stanford Linear Accelerator Center (SLAC), 2575 Sand Hill Road, Menlo Park, California 94025, USA \\ ${ }^{4}$ Helmholtz, Institute Jena, Fröbelstieg 3, 07743 Jena, Germany \\ ${ }^{5}$ optiX fab GmbH, Hans-Knöll-Strasse 6, 07745 Jena, Germany \\ ${ }^{6}$ China Academy of Engineering Physics (CAEP), Mianyang, China \\ ${ }^{7}$ Lawrence Livermore National Laboratory, 7000 East Avenue, Livermore, California 94550, USA \\ ${ }^{8}$ Institut für Physik, Universität Rostock, 18051 Rostock, Germany \\ ${ }^{9}$ Centre for Fusion, Space and Astrophysics, Department of Physics, University of Warwick, Coventry CV4 7AL, \\ United Kingdom \\ ${ }^{10}$ Institute of Applied Physics, Friedrich-Schiller University Jena, Albert-Einstein-Strasse 15, 07745 Jena, \\ Germany \\ ${ }^{11}$ The Hamburg Centre for Ultrafast Imaging CUI, Luruper Chaussee 149, 22761 Hamburg, Germany \\ ${ }^{12}$ Department of Physics, Clarendon Laboratory, University of Oxford, Parks Road, Oxford OX1 3PU, \\ United Kingdom \\ ${ }^{13}$ Deutsches Elektronen-Synchrotron DESY, Notkestrasse 85, 22607 Hamburg, Germany \\ ${ }^{14}$ Physikalisches Institut, Westfälische Wilhelms-Universität, Wilhelm-Klemm-Str. 10, 48149 Münster, Germany \\ ${ }^{15}$ Department of Physics, Faculty of Science, Tanta University, Tanta 31527, Egypt
}

(Received 4 October 2017; accepted 14 January 2018; published online 5 February 2018)

\begin{abstract}
We present an extreme ultraviolet (EUV) microscope using a Schwarzschild objective which is optimized for single-shot sub-micrometer imaging of laser-plasma targets. The microscope has been designed and constructed for imaging the scattering from an EUV-heated solid-density hydrogen jet. Imaging of a cryogenic hydrogen target was demonstrated using single pulses of the free-electron laser in Hamburg (FLASH) free-electron laser at a wavelength of $13.5 \mathrm{~nm}$. In a single exposure, we observe a hydrogen jet with ice fragments with a spatial resolution in the sub-micrometer range. In situ EUV imaging is expected to enable novel experimental capabilities for warm dense matter studies of micrometer-sized samples in laser-plasma experiments. Published by AIP Publishing. https://doi.org/10.1063/1.5007950
\end{abstract}

\section{INTRODUCTION}

Strongly correlated dense plasmas may be created by exciting a solid with sub-picosecond, high-intensity laser pulses. ${ }^{1}$ In the last decade, improvements in laser technology increased the potential repetition rate of such experiments to several shots per second. ${ }^{2}$ With each laser-matter interaction requiring a fresh target, this calls for high repetition-rate target delivery such as micrometer-thin liquid or cryogenic jets. Recently, it has been demonstrated that cryogenic hydrogen jets ${ }^{3}$ allow for studies of electron-ion equilibration ${ }^{4}$ and the generation of pulsed proton beams. ${ }^{5-7}$

The reliable shot-to-shot characterization of jet thickness, pointing jitter, relative alignment with tiny laser foci and potentially the time-resolved probing of the hydrodynamic plasma expansion, calls for high-resolution

a)ulf.zastrau@xfel.eu single-shot imaging with sub-micrometer resolution. Static, time-integrating microscopy in the sub- $\mu \mathrm{m}$ regime has already demonstrated the advantage of extreme ultraviolet (EUV) radiation using transmissive ${ }^{8}$ and reflective optics. ${ }^{9}$ Single-shot sensitivity can be achieved using large aperture optics with reflective multilayer coatings that have a reflectivity $R>65 \%$ with a spectral bandwidth of a few percent. An all-reflective optical system with high spatial resolution and good aberration characteristics is a Schwarzschild objective (SO) that employs two concentric spherical mirrors. ${ }^{10}$ A generic schematic of a Schwarzschild objective is shown in Fig. 1.

Intense, prominent examples of short-pulse sources of EUV radiation are, amongst others, high-harmonic generation from gases ${ }^{11}$ or surfaces ${ }^{12}$ and high-gain free-electron lasers (FELs). ${ }^{13}$ The latter provide sub-picosecond pulses with laserlike properties and about $10^{12}$ photons per pulse. Meanwhile, first high repetition rate studies of dense plasmas at the Freeelectron LASer in Hamburg (FLASH) were performed using jets from liquid water ${ }^{14}$ and cryogenic hydrogen. ${ }^{4,15}$ 


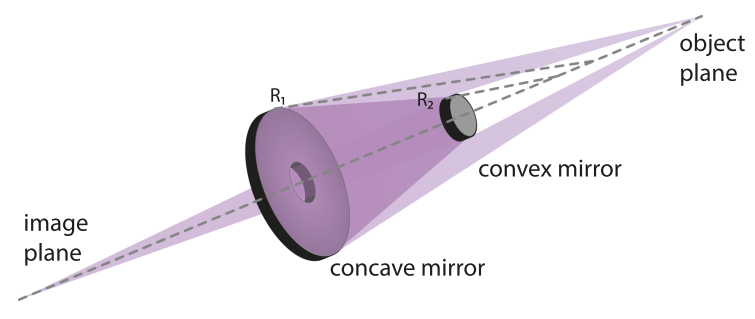

FIG. 1. Schematics of a Schwarzschild objective.

We have developed an experimental platform at FLASH for studying the dynamic properties of warm dense hydrogen, ${ }^{16-18}$ which allows us to investigate conditions typically found in planetary and fusion science. The basis for these studies is a cryogenically cooled hydrogen jet that liquefies hydrogen gas through a cryostat $(18 \mathrm{~K})$ and a circular nozzle (2-10 $\mu$ m diameter). ${ }^{3}$ The liquid hydrogen jet becomes solid due to evaporative cooling providing a cylindrically shaped target with a mass density of $\sim 0.08 \mathrm{~g} / \mathrm{cm}^{3}$. The intense EUV radiation ionizes the solid hydrogen target so that a solid density plasma is created. Even if fully ionized, the free electrons would result in a plasma frequency which is one order of magnitude lower than the frequency of $13.5 \mathrm{~nm}$ EUV photons (photon energy of $92 \mathrm{eV}$ ). Accordingly, EUV radiation can propagate into the plasma volume, in contrast to optical radiation in the visible or near-infrared spectrum which launches density waves. ${ }^{19}$ With an absorption length of $9 \mu \mathrm{m}$ for the $13.5 \mathrm{~nm}$ EUV radiation in our experiments, the EUV pulses are sufficiently absorbed and isochorically heat the cryogenic hydrogen target by photo ionization. We have recently shown that microscopic plasma properties can be investigated by EUV scattering: the elastic scattering cross section increases by more than a factor of two when solid density hydrogen dissociates and becomes ionized as a result of electron-ion heat transfer. ${ }^{4}$ These results impact the modeling of planetary systems and inertial confinement fusion via the measurements of the dynamics of plasma parameters. These measurements so far average over the nearly Gaussian intensity distribution of the laser focus. Accordingly, the lack of spatial resolution limits the accuracy to which the applied intensity can be determined. For the experimental platform at FLASH, where the focal spot size of 25-30 $\mu \mathrm{m}$ could limit the spatial resolution, an EUV imaging system with $\sim 1 \mu \mathrm{m}$ spatial resolution would be able to resolve the scattered radiation from sites with defined irradiation intensity. Moreover, imaging the scattering objects and the structure of the hydrogen target for each laser pulse would allow a more detailed analysis, e.g., target characterization or the disentanglement of volume and surface scattering. The development of an EUV microscope for these experiments thus significantly improves the experimental capabilities for studying dense plasmas at EUV free-electron lasers.

Here we present an EUV microscope based on a Schwarzschild objective. We demonstrate its high spatial resolution and detection efficiency by observing single-shot scattering from a weakly scattering cryogenic hydrogen jet at the FLASH FEL.

\section{DESIGN CONSIDERATIONS OF THE SCHWARZSCHILD OBJECTIVE AND THE EUV MICROSCOPE}

In the Schwarzschild geometry, the degrees of freedom for the radius of curvature of the two spherical mirrors, and therefore also the distances, are determined by the magnification $M$. Larger mirrors increase the numerical aperture and shrink the Airy disc. However, aberrations from the outer parts of the mirror will compromise the resolution. This could be compensated, for example, by an aspherical surface of the small mirror. We based our design on an optimized, compact design which was initially published by Artioukov and Krymski in $1999^{10}$ and was frequently used thereafter. ${ }^{20,21} \mathrm{We}$ found that this design is a good compromise in terms of optics size, resolution, and aberration mitigation.

We performed a detailed design study using the raytracing software ZEMAX. Simulations confirmed that the initial parameters lead to optimum aberration correction. A tolerance analysis has also shown that the performance of the SO is relatively robust regarding misalignment and manufacturing tolerances. The optimization with ZEMAX has shown that the magnification can be controlled over a large parameter range with excellent aberration characteristics using the same optical elements. The parameters of the optical elements of the SO are summarized in Table I. Before different options in terms of resolution and magnification are presented, we discuss general design criteria of the EUV microscope with respect to the experiment.

The requirements in terms of magnification are driven by the experiment and the detector: In the case of the hydrogen jet, a spatial resolution of $\leq 1 \mu \mathrm{m}$ is needed in order to resolve the jet diameter of $2-10 \mu \mathrm{m}$ and to discriminate between different intensity regimes across the FEL focus with a spot size of 25-30 $\mu \mathrm{m}$ full-width at half-maximum (FWHM). Since the smallest pixel pitch of commercially available direct-detection EUV CCDs is $13.5 \mu \mathrm{m}$, the required magnification of the

TABLE I. Parameters of the Schwarzschild mirrors following the design by Artioukov and Krymski. ${ }^{10}$ The tolerances relate to the substrate specification used for the microscope in this publication. For the surface accuracy, we assume $\lambda=500 \mathrm{~nm}$.

\begin{tabular}{ll}
\hline MIRROR 1-concave & \\
\hline Large mirror diameter $D_{1}(\mathrm{~mm})$ & $50(-0.1)$ \\
Thickness at edge $d(\mathrm{~mm})$ & $9.5( \pm 0.1)$ \\
Radius of curvature $(\mathrm{RoC}) R_{1}$ & $-100 \mathrm{~mm}( \pm 0.5 \%)$ \\
RoC error & $\pm 1 \%$ \\
Hole diameter in large mirror $(\mathrm{mm})$ & $10.8( \pm 0.1)$ \\
Surface accuracy $\eta$ & $\lambda / 30($ within $95 \%$ of the area) \\
Surface roughness $\sigma$ & $<0.25 \mathrm{~nm} \mathrm{rms}$ \\
\hline MIRROR 2-convex & \\
\hline Small mirror diameter $D_{2}(\mathrm{~mm})$ & 10.6 \\
Thickness in center $d(\mathrm{~mm})$ & $6.35( \pm 0.1)$ \\
Radius of curvature $(\mathrm{RoC}) R_{2}$ & $35 \mathrm{~mm}( \pm 0.5 \%)$ \\
RoC error $(\%)$ & \pm 1 \\
Surface accuracy $\eta$ & $\lambda / 30($ within $95 \%$ of the area) \\
Surface roughness $\sigma$ & $<0.25 \mathrm{~nm} \mathrm{rms}$ \\
\end{tabular}


microscope should be larger than $>13.5 \times$ to allow for imaging $1 \mu \mathrm{m}$ structures with a standard EUV CCD.

The SO design described in Ref. 10 has a magnification $M$ of 21.27 which would allow us to resolve sub $\mu$ m structures with the CCD. For this setup, simulations using ZEMAX yield an Airy disk diameter in the detector plane of $1.84 \mu \mathrm{m}$ and an aberrated geometric point spread function (PSF) of $2 \mu \mathrm{m}$. This is in agreement with previously published analytic results ${ }^{10}$ and corresponds to a spatial resolution of $2 \mu \mathrm{m} / M \sim 100 \mathrm{~nm}$. However, the achievable resolution is limited by the CCD pixel size which can be calculated to $13.5 \mu \mathrm{m} / M \sim 0.6 \mu \mathrm{m}$ for this configuration.

For different magnification settings, the distances between the CCD detector and the two spherical mirrors must be adapted. Table II lists magnifications for mirror distances which are varied in $100 \mu \mathrm{m}$ steps. The corresponding parameters have been optimized using ZEMAX. At a magnification of $\sim 89$, the Airy spot and the pixel size of $13.5 \mu \mathrm{m}$ coincide and therefore the highest spatial resolution of $\sim 150 \mathrm{~nm}$ can be achieved. However, the field of view recorded by the CCD camera will be very small and the CCD has to be placed $2.4 \mathrm{~m}$ away from the objective. Such a high spatial resolution is also not needed for our experiments. We therefore found a compromise by increasing the magnification slightly to $M=35$ as compared to Ref. 10 . The aberrations within the resulting $150 \mu \mathrm{m}$ field-of-view (FoV) of the jet-FEL interaction are still $<0.3 \mu \mathrm{m}$ and do not compromise the imaging quality of the presented EUV microscope. Outside the FoV, the image is strongly distorted.

Both mirrors are coated with a high-reflectivity molybdenum (Mo) and silicon ( $\mathrm{Si}$ ) multilayer for a center wavelength of $13.5 \mathrm{~nm}$. The spectral bandwidth amounts to $\sim 0.5 \mathrm{~nm}$ FWHM but is narrowed to about $0.2 \mathrm{~nm}$ due to the two subsequent reflections. The coating is optimized for incidence angles between $1^{\circ}-8^{\circ}$ and has a reflectivity of $R \sim 0.68$. This yields a throughput of $46 \%$ of the optical system. The angle variation over the mirror aperture still allows for a homogeneous coating, no gradient in the multilayer spacing is required. We also note that the changes of the incidence angle for the different magnification settings as presented in Table II are small compared to the angular coverage of the reflection curve, i.e., one multilayer coating can be used for all presented magnification settings.

Another important design consideration is the efficiency of the optical system since we would like to obtain microscopy images with a single FEL pulse. Owing to fluctuations of the hydrogen jet target and the EUV source, single-exposure imaging (requiring $10^{3}-10^{4}$ photons per image) is crucial for successful experiments. The detection efficiency $\eta$ of the presented design, defined as the number of detected photon per isotropically emitted source photon, can be estimated by

$$
\begin{aligned}
\eta & =\frac{1}{4 \pi}\left(\frac{A_{1}}{d_{1}^{2}}-\frac{A_{2}}{d_{2}^{2}}\right) R^{2} \eta_{\mathrm{QE}}(\lambda) \\
& =4.5 \times 10^{-3} \times 0.46 \times 0.45=9 \times 10^{-4},
\end{aligned}
$$

where $A_{1}$ is the surface area of the large concave mirror at distance $d_{1}$ from the source point, $A_{2}$ is the area of the small convex mirror mount at distance $d_{2}$ which will partly obscure the large mirror, $R \sim 0.68$ is the reflectivity of a single multilayer mirror, and $\eta_{\mathrm{QE}}(\lambda)$ is the quantum efficiency of the detector (Princeton Instruments PIXIS-XO: 2KBTM back thinned CCD).

For the hydrogen jet experiment, we assume $10^{12}$ FLASH photons per pulse in the focus, an incoherently scattered fraction of $\sim 10^{-5}$ into the full solid angle and hence $10^{7}$ scattered photons. Applying the above-estimated $\eta$, this will yield images containing $\leq 10^{4}$ photons per single exposure. If the imaged object covers $\sim 100$ pixels, each pixel would contain on average $\sim 100$ photons.

We assess the influence of misalignment on the spatial resolution using ZEMAX simulations. If the mirrors were laterally misaligned by $\Delta x \leq \pm 15 \mu \mathrm{m}$ with respect to each other, the calculated PSF on the optical axis is $400 \mathrm{~nm}$. If the centers of curvature of the two mirrors were separated by $\Delta Z \leq$ $\pm 500 \mu \mathrm{m}$ along the optical axis and the image is refocused by adjusting the distance of the microscope to the target, a PSF of $\leq 1 \mu \mathrm{m}$ can be maintained. Another issue is the misalignment in the angle of the spherical mirrors. Assuming an angular misalignment or tolerance of the mirror substrate of $>5$ arc min, this would result in an aberrated PSF in the detector plane of $6.7 \mu \mathrm{m}$, i.e., half a pixel pitch.

From the analysis of alignment requirements, we decided to develop a simple mechanical system for mounting the optics. Fine-precision machining of the mount components should be sufficient in order to fulfill the rather relaxed requirements in terms of resolution and alignment precision. We decided to build a mechanically robust EUV microscope system that can be added to an experimental platform in a flexible way without elaborate alignment procedures. We therefore constructed a mount system with a minimum amount of motions. This also limits the coupling of vibrations of the

TABLE II. Parameters of the EUV microscope using different magnification settings at a wavelength of $\lambda=13.5 \mathrm{~nm}$. The half-width at half-maximum of point spread function (PSF) due to spherical aberration amounts

\begin{tabular}{|c|c|c|c|c|c|c|}
\hline Magnification, $M$ & 21.27 & 26.27 & 34.34 & 49.55 & 88.94 & 433.64 \\
\hline Axial distance between mirrors (mm) & 65.0 & 64.9 & 64.8 & 64.7 & 64.6 & 64.5 \\
\hline Distance convex-mirror-to-detector (mm) & 564.68 & 701.47 & 922.22 & 1338.50 & 2416.57 & $\approx 11850$ \\
\hline Airy disk radius in detector plane $(\mu \mathrm{m})$ & 1.84 & 2.28 & 2.98 & 4.30 & 7.73 & 38.08 \\
\hline PSF in detector plane, $2 \Delta x(\mu \mathrm{m})$ & 1.94 & 2.81 & 4.17 & 6.70 & 13.31 & 70.37 \\
\hline Maximum spatial resolution, $\Delta x / M(\mathrm{~nm})$ & $\approx 45$ & $\approx 53$ & $\approx 61$ & $\approx 68$ & $\approx 75$ & $\approx 81$ \\
\hline CCD-pixel-limited resolution, $13.5 \mu \mathrm{m} / M(\mathrm{~nm})$ & 635 & 514 & 393 & 272 & 148 & 31 \\
\hline FoV for $1^{\prime \prime} \mathrm{CCD}(\mu \mathrm{m})$ & 1300 & 1053 & 805 & 557 & 303 & 63 \\
\hline
\end{tabular}
to $\Delta x$. 

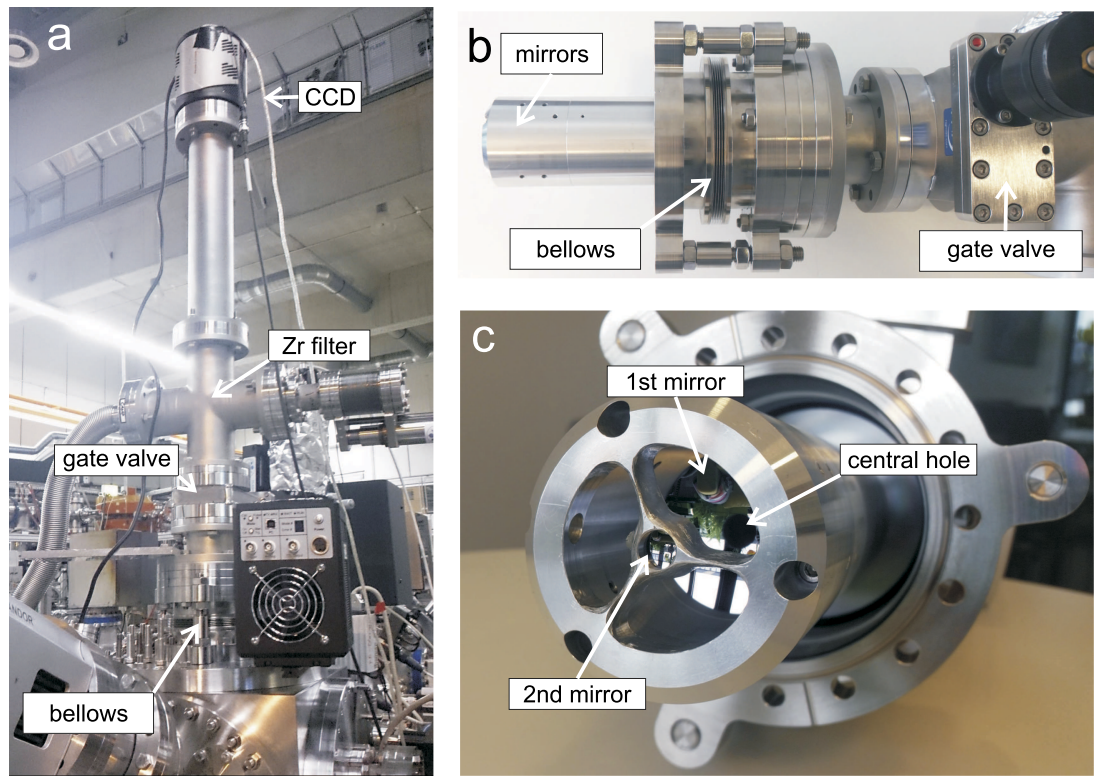

FIG. 2. Three photographs of the EUV Schwarzschild microscope. (a) The EUV microscope mounted on top of a vacuum chamber at the FLASH freeelectron laser. (b) A side view of the assembly. (c) A view of the two multilayer mirrors in their mount.

mount system to the optical components. Some photographs of the vacuum housing and the EUV microscope are shown in Figs. 2(a)-2(c).

The distance between the CCD detector and the mirror assembly determines the magnification, and the length of the vacuum tube is adapted accordingly. In order to obtain sharp images on the detector, at least one of the distances source$S O$, concave-convex mirror, and SO-detector plane has to be adjustable during the experiment. We used bellows with manual position control to both steer the image to the center of the CCD detector and to adjust the distance between source and the objective. In order to separate the (potentially cooled) CCD detector from the source in the interaction vacuum chamber, we installed a gate valve between the SO and the CCD. This allows us to vent the interaction chamber while keeping the detector cooled under vacuum conditions. Since the SO also focuses optical light from the source point, we can insert a $200 \mathrm{~nm}$ thin zirconium ( $\mathrm{Zr}$ ) foil using a vacuum manipulator to block optical light. The transmission of $13.5 \mathrm{~nm}$ photons through the foil is $50 \%$.

\section{CALIBRATION USING FIDUCIAL GRIDS}

The EUV microscope was mounted to the experimental platform at FLASH. EUV pulses with a wavelength of $13.5 \mathrm{~nm}$, a pulse duration of $60 \mathrm{fs}$, and a pulse energy of $\sim 150 \mu \mathrm{J}$ were focused to the center of the vacuum chamber where test targets and the hydrogen jet can be inserted. The EUV microscope is vertically oriented so that the EUV pulses are scattered approximately $90^{\circ}$ into the EUV microscope. Fiducial grids were used for calibrating the EUV microscope. The plane of the metallic grids were oriented with an angle of $\sim 30^{\circ}$ to the focal plane to allow for EUV illumination with the FLASH facility. We aligned the EUV microscope manually so that a sharp image was achieved. The results are shown in Fig. 3. A magnification of $M=32.7$ was found, which means that a $13.5 \mu \mathrm{m}$ pixel pitch corresponds to $0.41 \mu \mathrm{m}$ in the object plane. This is very close to the design value and implies that the spherical mirrors are a few micrometers closer than anticipated. This is well within the tolerances of the machining precision. A FoV of $100-150 \mu \mathrm{m}$ was demonstrated using the alignment grids.
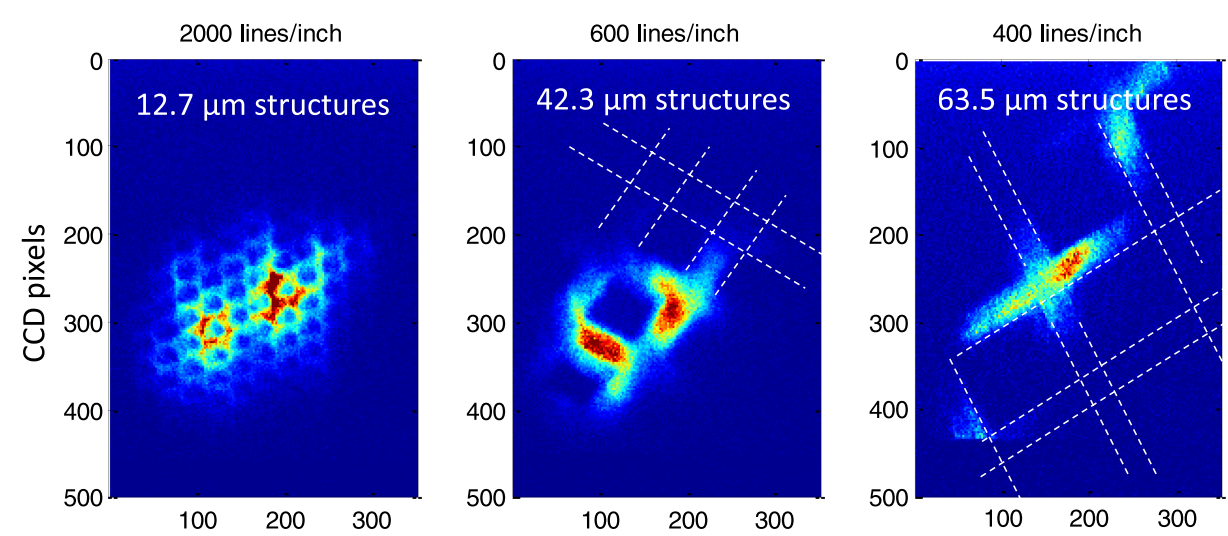

FIG. 3. SO images of single-shot exposures of three calibration grids with different spacing. The dashed white lines are guides to the eye and resemble the grid structure. 


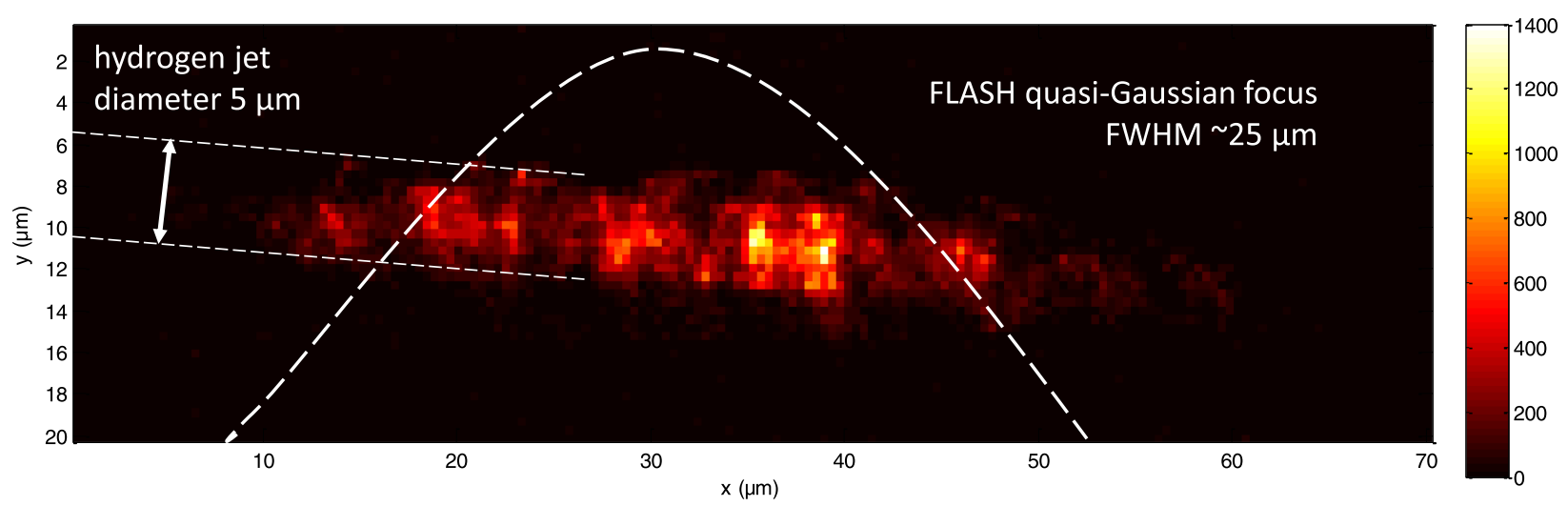

FIG. 4. Typical EUV image of the hydrogen target using a single EUV pulse from FLASH. As indicated by the dashed white cure, the EUV pulses are focused to $25 \mu \mathrm{m}$ (FWHM) to a peak intensity of $\sim 10^{14} \mathrm{~W} / \mathrm{cm}^{2}$ and scatter of the hydrogen target. The $5 \mu \mathrm{m}$ jet diameter can be resolved. The inhomogeneous ice structure of the hydrogen jet can be observed in single exposure EUV imaging.

\section{EUV MICROSCOPY OF WARM DENSE HYDROGEN PLASMAS USING A SCHWARZSCHILD OBJECTIVE AT FLASH}

For generating warm dense hydrogen plasmas, we used the cryogenic jet with a diameter of $5 \mu \mathrm{m}$. It was irradiated by individual FLASH pulses and images were recorded with a repetition rate of $10 \mathrm{~Hz}$ using the EUV microscope. Figure 4 shows a typical image of the initial hydrogen ice jet, illuminated in a $\sim 50 \mu \mathrm{m}$ wide region by a nearly Gaussian shaped EUV focus of $25 \mu \mathrm{m}$. Most importantly, our experiment shows that quantitative single-pulse scattering data can be recorded using the EUV microscope. This novel experimental capability will allow scattering experiments with improved data analysis and high statistical significance. For example, we can record the intensity distribution of the FLASH focus on-target and subsequently analyze scattering from selected intensity intervals. Moreover, we can image the longitudinal structure of the jet target: We find that the jet structure varies from shot-to-shot and deviates from the assumed cylindrical shape. Apparently, ice fragments and crystallization formation strongly influence the geometry of the target which has to be accounted for in the analysis of the experiments.

We observe small ice fragments in many of our images. An example, which shows a very small hydrogen ice fragment is shown in Fig. 5. The imaged ice fragment has a size of $\sim 2$ pixels (FWHM) or $\sim 0.8 \mu \mathrm{m}$. Accordingly, the small ice fragment can be used to estimate the upper limit for the spatial resolution. This example demonstrates that we can achieve sub-micrometer EUV imaging of hydrogen targets with a single FLASH pulse. We have ruled out that the observed structures originate from the intensity distribution within the

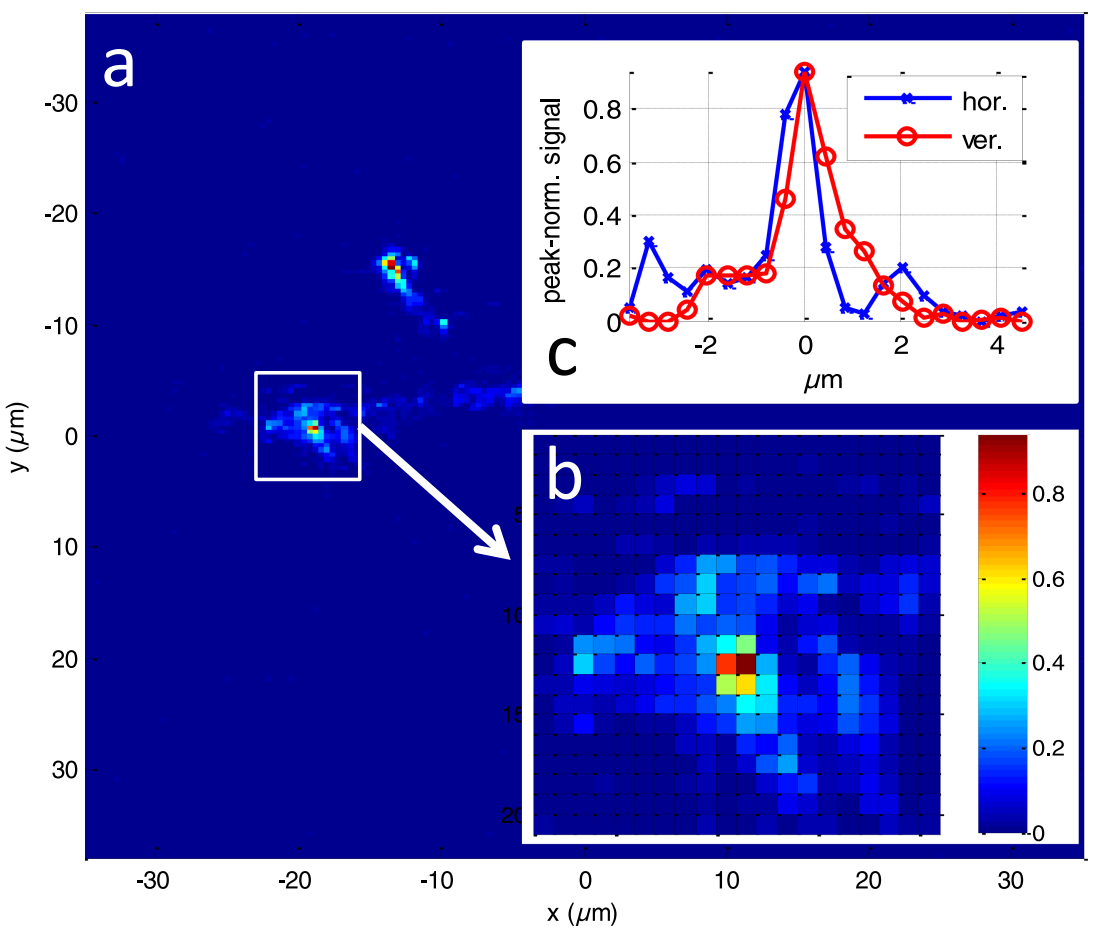

FIG. 5. Single-shot scattering from nanometer-scale hydrogen ice fragments. The CCD image (a) shows two fragments which are parts of the hydrogen target. For illustrating the spatial resolution of the EUV microscope, a magnified region is shown in inset (b). The horizontal and vertical profiles in inset (c) demonstrate a spatial resolution $\lesssim 1 \mu \mathrm{m}$. 
FEL focus by illuminating samples with homogeneous density and smooth surface. In an EUV-pump EUV-probe experiment, the amount of scattered radiation will rise by more than a factor of two, ${ }^{4}$ as compared to the single exposure reported here.

\section{CONCLUSION AND OUTLOOK}

We have demonstrated an EUV microscope for scattering experiments at the FLASH free-electron laser using a Schwarzschild objective. The design of the EUV microscope allows us to perform sub-micrometer resolved imaging of solid-density hydrogen targets. We were able to acquire images with single pulse exposure using FLASH pulses with a wavelength of $13.5 \mathrm{~nm}$ and a pulse energy of $150 \mu \mathrm{J}$. The EUV microscope has a robust mechanical design and can easily be integrated to the experimental platform. These novel imaging capabilities will allow improved experimental studies of EUV heated hydrogen plasmas. For example, single pulse imaging data facilitates intensity-resolved scattering experiments. Moreover, imaging the hydrogen jet with single exposure has revealed the complex geometry of the target that needs to be taken into account in the analysis of the experiments. Further, it might open interesting scientific questions in the formation and crystallization of hydrogen.

More generally, the ability to characterize the micrometersized laser-target interaction with sub-picosecond time resolution in the EUV has a wide application range. For example, $68 \mathrm{eV}$ extreme ultraviolet (XUV) plasma emission was employed to infer the plasma temperature, ${ }^{22}$ but this study was limited to a spatial resolution of $\sim 10 \mu \mathrm{m}$. It becomes possible to perform these studies with sub- $\mu$ m resolution, yielding information on electron transport and filamentation. Alternatively, choosing the monochromatic reflectivity of the multilayer to match a specific emission line will enable us to resolve transport processes such as ionization fronts or heat flux in mass limited targets, in particular with anisotropic targets such as liquid jets, droplets, and nano-wires.

\section{ACKNOWLEDGMENTS}

This work was partially supported by the German Ministry for Education and Research (BMBF) via a Priority Program (Nos. FSP 301 and 302). C. Rödel acknowledges support from the Volkswagen Foundation. The work of T.D. was performed under the auspices of the U.S. Department of
Energy by Lawrence Livermore National Laboratory under Contract No. DE-AC52-07NA27344. The SLAC HED group is supported by Fusion Energy Sciences under FWP100182. C.F.G. acknowledges support from the European Cluster of Advanced Laser Light Sources (EUCALL) project which has received funding from the European Union's Horizon 2020 research and innovation programme under grant agreement No. 654220.

${ }^{1}$ M. Nakatsutsumi, K. Appel, C. Baehtz, B. Chen, T. Cowan, S. Göde, Z. Konopkova, A. Pelka, G. Priebe, A. Schmidt et al., Plasma Phys. Controlled Fusion 59, 014028 (2016).

${ }^{2}$ M. Gauthier, L. Fletcher, A. Ravasio, E. Galtier, E. Gamboa, E. Granados, J. Hastings, P. Heimann, H. Lee, B. Nagler et al., Rev. Sci. Instrum. 85, 11 E616 (2014).

${ }^{3}$ J. Kim, S. Göde, and S. Glenzer, Rev. Sci. Instrum. 87, 11E328 (2016).

${ }^{4}$ U. Zastrau, P. Sperling, M. Harmand et al., Phys. Rev. Lett. 112, 105002 (2014).

${ }^{5}$ S. Göde, C. Rödel, K. Zeil, R. Mishra, M. Gauthier, F.-E. Brack, T. Kluge, M. MacDonald, J. Metzkes, L. Obst et al., Phys. Rev. Lett. 118, 194801 (2017).

${ }^{6}$ L. Obst, S. Göde, M. Rehwald, F.-E. Brack, J. Branco, S. Bock, M. Bussmann, T. E. Cowan, C. B. Curry, F. Fiuza et al., Sci. Rep. 7, 10248 (2017).

${ }^{7}$ M. Gauthier, C. Curry, S. Göde, F.-E. Brack, J. Kim, M. MacDonald, J. Metzkes, L. Obst, M. Rehwald, C. Rödel et al., Appl. Phys. Lett. 111, 114102 (2017).

${ }^{8}$ P. W. Wachulak, A. Bartnik, H. Fiedorowicz, and J. Kostecki, Opt. Express 19, 9541 (2011).

${ }^{9}$ J. Ewald, M. Wieland, T. Nisius, L. Henning, T. Feigl, M. Drescher, and T. Wilhein, J. Phys.: Conf. Ser. 499, 012008 (2014).

${ }^{10}$ I. A. Artioukov and K. M. Krymski, Optical Eng. 39, 2163 (2000).

${ }^{11}$ P. M. Paul, E. Toma, P. Breger, G. Mullot, F. Augé, P. Balcou, H. Muller, and P. Agostini, Science 292, 1689 (2001).

${ }^{12}$ C. Rödel, D. an der Brügge, J. Bierbach, M. Yeung, T. Hahn, B. Dromey, S. Herzer, S. Fuchs, A. G. Pour, E. Eckner et al., Phys. Rev. Lett. 109, 125002 (2012).

${ }^{13}$ W. Ackermann et al., Nat. Photonics 1, 336 (2007).

${ }^{14}$ J. Kim, P. Sperling, Z. Chen, S. Toleikis, C. Curry, M. Mo, R. Redmer, D. Daniel, and S. Glenzer, in APS Meeting Abstracts, 2016.

${ }^{15}$ R. R. Fäustlin et al., Phys. Rev. Lett. 104, 125002 (2010).

${ }^{16}$ A. Höll, T. Bornath et al., High Energy Density Phys. 3, 120 (2007).

${ }^{17}$ S. Toleikis, R. R. Fäustlin et al., High Energy Density Phys. 6, 15 (2010).

${ }^{18} \mathrm{~S}$. Toleikis, T. Bornath, T. Döppner et al., J. Phys. B: At., Mol. Opt. Phys. 43, 194017 (2010).

${ }^{19}$ M. Shihab, T. Bornath, and R. Redmer, Plasma Phys. Controlled Fusion 59, 045006 (2017).

${ }^{20}$ F. Barkusky, C. Peth, K. Mann, T. Feigl, and N. Kaiser, Rev. Sci. Instrum. 76, 105102 (2005).

${ }^{21}$ L. Juschkin, A. Maryasov, S. Herbert, A. Aretz, K. Bergmann, and R. Lebert, in The 10th International Conference on X-ray Microscopy (AIP Publishing, 2011), Vol. 1365, pp. 265-268.

${ }^{22}$ P. Gu, B. Zhang, M. Key, S. Hatchett, T. Barbee, R. Freeman, K. Akli, D. Hey, J. King, A. Mackinnon et al., Rev. Sci. Instrum. 77, 113101 (2006). 